\title{
Gold Nanoparticle and Berberine Entrapped into Hydrogel Matrix as Drug Delivery System
}

\author{
Camila Rufino Souza', Henrique R. Oliveira', Wagner M. Pinheiro², \\ Lubhandwa S. Biswaro², Ricardo B. Azevedo ${ }^{2}$, Anderson J. Gomes ${ }^{1 *}$, Claure N. Lunardi ${ }^{*}$ \\ ${ }^{1}$ Laboratory of Nanobiotechnology, Faculty of Ceilandia, University of Brasilia, Brasilia, Brazil \\ ${ }^{2}$ Institute of Biological Sciences, University of Brasilia, Brasilia, Brazil \\ Email: ${ }^{*}$ clunardi@unb.br, ${ }^{*}$ ajgomes@unb.br
}

Received 16 December 2014; accepted 29 December 2014; published 14 January 2015

Copyright (C) 2015 by authors and Scientific Research Publishing Inc.

This work is licensed under the Creative Commons Attribution International License (CC BY). http://creativecommons.org/licenses/by/4.0/

\section{Open Access}

\section{Abstract}

In this study the novel hydrogel loaded with gold nanoparticle (AuNP) enhanced the berberine (BS) release when compared with other formulations of hydrogel. Hydrogels are hydrophilic polymer networks having the capacity to absorb water, ranging from about twenty to thousand times their dry weight. BS is a natural product, a quaternary ammonium salt from the group of isoquinoline alkaloids found in medicinal plants as Berberis Vulgaris. BS has some activity against dysentery, hypertension, inflammation, and liver disease in China and Japan. In this work, BS was used as a model drug to study its association with different types of hydrogel composites of polyvinyl alcohol (BS-PVA 10\%); gellan gum (BS-GG 2\%), gellan gum-PVA crosslinked with cysteine (cys) (BSGG2\%PVA2\%cys) and gellan gum-PVA cosslinked with cysteine associated with gold nanoparticles (AuNP-BSGG2\%PVA2\%cys). Several parameters such as fraction of retained water (Wf), hydration percentage $(\% \mathrm{H})$, Swelling (DSw) and time course profile $(t=100 \%)(\mathrm{TC})$ were evaluated for all preparations. The results showed that the AuNP-BS-GG2\%PVA2\%cys was able to retain more water and swelling than the other preparations. The time course of release of the $B S$ to the medium was greater for AuNP-BS-GG2\%PVA2\%cys making it a candidate to drug delivery studies in biological assays. Also Scanning Electron Microscopy (SEM) images of the surface of these hydrogel were performed. Furthermore, crosslink of the resulting hydrogels were investigated by Fourier Transform Infrared Spectroscopy (FTIR) and differential scanning calorimetry (DSC) and thermogravimetric analysis (TGA). Thus, briefly, the aim of this work was to study three composition of hydrogel loaded with BS and its composition in relation to addition to AuNP and evaluate its profile for further drug delivery application using the Surface Plasmonic Resonance (SPR) as a tool improving the drug release in the new hydrogel.

\footnotetext{
${ }^{*}$ Corresponding authors.

How to cite this paper: Rufino Souza, C., Oliveira, H.R., Pinheiro, W.M., Biswaro, L.S., Azevedo, R.B., Gomes, A.J. and Lunardi, C.N. (2015) Gold Nanoparticle and Berberine Entrapped into Hydrogel Matrix as Drug Delivery System. Journal of Biomaterials and Nanobiotechnology, 6, 53-63. http://dx.doi.org/10.4236/jbnb.2015.61006
} 


\section{Keywords}

\section{Hydrogel, Berberine, Drug Delivery, AuNPs}

\section{Introduction}

Berberine (BS) is a natural product, a quaternary ammonium salt from the group of isoquinoline alkaloids, belongsto structural class of protoberberine, which positively-charged ion turns it hydro soluble. It is found in medicinal plants as Berberis Vulgaris [1]-[3] and the salts are derived of ammoniac form, solid, nonvolatile, crystallizable and inodorous when it is oxygenized. Due to its yellow color and characteristics the BS is applied with basic nature pigment [4]. The most interesting aspect of BS is related to its pharmacological properties been reported in the treatment of illness, such as: dysentery, hypertension, diabetes, inflammation, and also cancer [5][12].

Hydrogel is report itself as polymeric network (reticulated structure), which has the feature to swell with water or biological fluids. Moreover, hydrogel storage a large amount of these fluids in its structure without dissolves itself [13] [14]. Besides, it exhibits some particular characteristics, such as hydrophilic behavior, insoluble in water, floppy, elastic and in contact with water, its volume increases considerably, without lose its shape, until achieve physic-chemical equilibrium. Hydrogels are able to mimic nature tissue, being biocompatibility [15] [16]. This feature leads to the amount of storage a large amount of water and special surface property. Hence, in this background, synthetics hydrogels are awaken interest at tissue engineering, cell encapsulate and drug delivery [17]. Another type of nanomaterial that have attracted increasing attention due to their unique properties in multidisciplinary research fields are gold nanoparticles (AuNPs) [2] [3]. The gold core is inert and essentially non-toxic to cells [18]. The particles absorb and resonantly scatter visible and near-infrared light upon excitation of their surface plasmon oscillation (SPR).

Gold nanoparticles associated to hydrogels are based on the behavior of AuNPs properties like the SPR band leading to evaluation of parameters like size among others [19]. Another important characteristic of AuNPs is
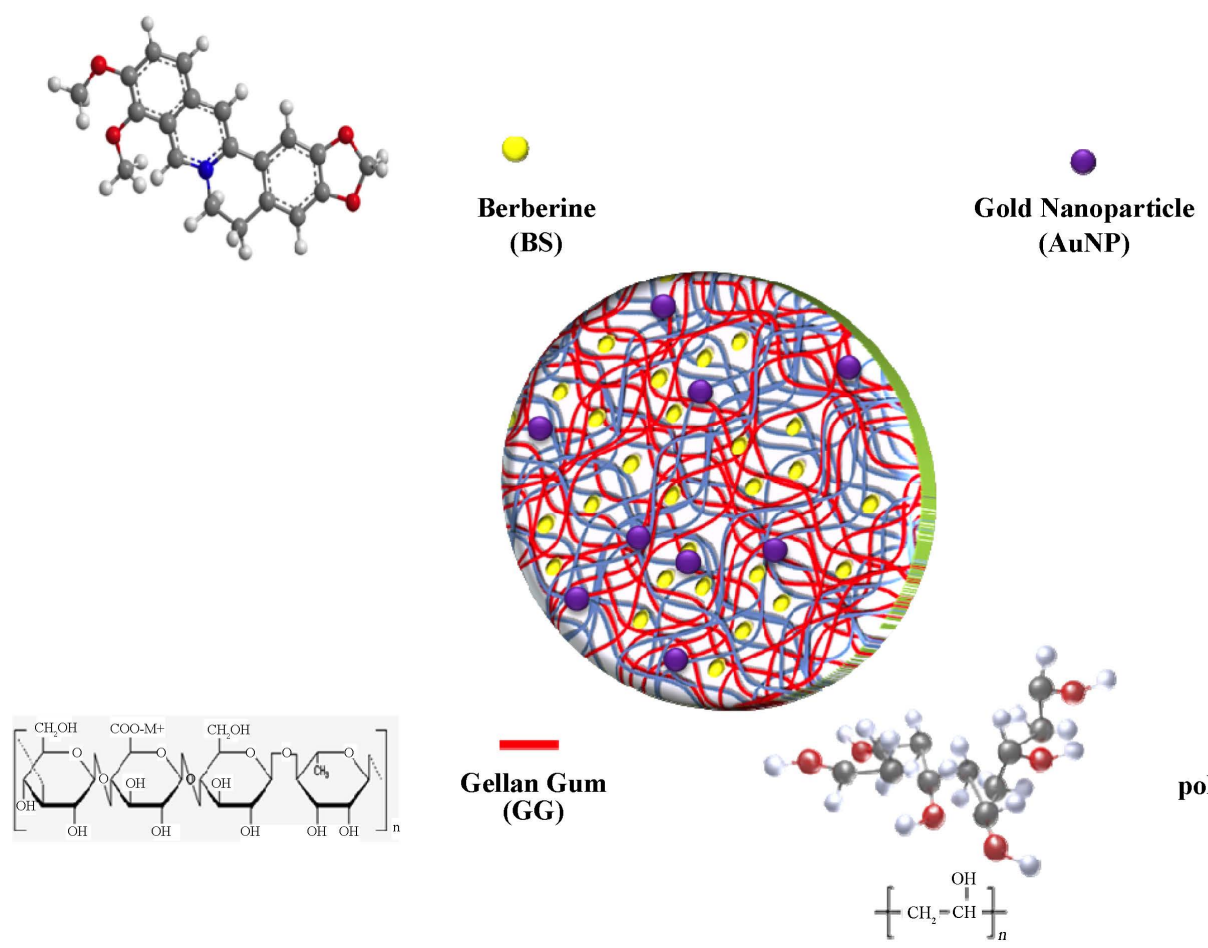

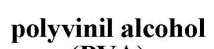

(PVA)

Figure 1. Schematic illustration of possible interaction of BS-GG2\%PVA2\%cys and AuNPs into the composite. 
the ability of dissipating heat when absorbing light, being an interest approach for thermal therapy as a trigger to release of encapsulated drugs from thermosensitive hydrogels [9] [17]. Moreover, there are no reports on the use of BS for preparing crosslinked AuNPs hydrogel nanocomposites, despite its relevance in the context of new formulations for biocompatible hydrogels. The aim of this article was to study the different types of hydrogelbiodegradable composites and the inclusion of gold nanoparticles to this system, evaluating parameters of surface and it's correlation to release time course profile of BS improving the efficiency and versatility to be used in existing therapies.

\section{Experimental}

\subsection{Materials}

Berberine (BS from Sigma Chem. Co.); polyvinil alcohol (88\% PVA from Sigma Chem. Co.); Deacylated gellan gum GG-LA (Kelcogel from Cpkelco); chloridric acid (from Akros Chem); Au(III) solution (1\%) was prepared with HAuCl4, citric acid (from Sigma Chem. Co.); HEPES buffer (from Sigma Chem. Co.); Cisteyne (CYS). Double distilled water was used for the preparation of all solutions.

\subsection{Preparation of Hydrogel Composites}

PVA 10\%. Hydrogel composites were prepared with 10\% PVA solution, 7.5\% citric acid solution in distilled water,BS was and used as received. All the reagents were of analytical grade. The preparation of the hydrogel has been described elsewhere [20]. In short, $100 \mathrm{~mL}$ of a 10\% aqueous solution of PVA and citric acid solution and concentrated hydrochloric acid $(\mathrm{HCl}, 0.05 \mathrm{~mL}$ ) were mixed, and the resulting dispersion was stirred (using a overhead stirrer at $100 \pm 5 \mathrm{rpm}$ ) at $70^{\circ} \mathrm{C}$ for a half-hour to carry out the crosslinked PVA hydrogel production. The thick dispersion so obtained was converted into a membrane by the conventional solution casting method. The resultant membrane was washed thoroughly with distilled water to remove the $\mathrm{HCl}$ and was stored in a desiccators at $37^{\circ} \mathrm{C}$. The empty hydrogel was designated PVA $10 \%$ and the berberine loaded was designated BSPVA10\%.

Gellan Gum. The gellan gum (GG) hydrogel composite were prepared using HEPES (50 mM, pH = 4), fol-

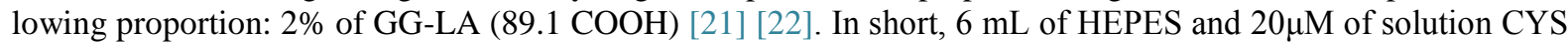
and GG-LA were mixed, and the resulting dispersion was stirred (using a overhead stirrer at $100 \pm 5 \mathrm{rpm}$ ) at $80^{\circ} \mathrm{C}$ for ten minutes to carry out the crosslinked kelcogell hydrogel production. The thick dispersion so obtained was converted into a membrane by the conventional solution casting method. The empty hydrogel was designated GG2\% and the berberine loaded was designated BS-GG2\%.

PVA 2\% plus Gellan Gum 2\%. The hydrogel composite were prepared using HEPES $50 \mathrm{mM}$, pH = 4), following proportion: 2\% of GG-LA (89.1 COOH) and 2\% of PVA. In short, $6 \mathrm{~mL}$ of HEPES, $20 \mu \mathrm{M}$ of solution CYS, GG-LA and PVA were mixed, and the resulting dispersion was stirred (using a overhead stirrer at $100 \pm 5$ $\mathrm{rpm}$ ) at $80^{\circ} \mathrm{C}$ for ten minutes to carry out the crosslinked kelcogell hydrogel production. The thick dispersion so obtained was converted into a membrane by the conventional solution casting method. The empty hydrogel was designated GG2\%PVA2\%cys and the berberine loaded was designated BS-GG2\%PVA2\%cys.

The BS in micromolar range $(10-6 \mathrm{M})$ concentration was added before stirring time to produce hydrogels composites. AuNPs: Gold nanoparticle was prepared by modified sodium citrate reduction method by Turkevich [23]. Placed $0.70 \mathrm{~mL}$ of $0.1 \%$ HAuCl 4 into a $150 \mathrm{~mL}$ beaker, then added water to near $60 \mathrm{~mL}$ and the solution was heated to boiling. An amount of $2 \mathrm{~mL}$ of $1 \%$ sodium citrate solution was added dropwhise into beaker solution. The solution became rubbish during stirring for 30 minutes. This solution is called naked AuNPs.

AuNPs-PVA 2\% plus Gellan Gum 2\%. The nanocomposites were prepared by blending the Au NPs with the PVA 2\% plus Gellan Gum 2\%. The colloidal AuNPs was added to the BS in micromolar range (10-6 M) concentration was added before stirring time to produce hydrogels composites in the same procedure as described before.

\subsection{In Vitro Stability and Swelling of Biodegradable Hydrogel Composite}

Approximately $30 \mathrm{mg}$ of each type of composites loaded with $465 \mu \mathrm{M}$ of BS was deposited into pre-weighed polypropylene sample tubes, weighed, warmed to $37^{\circ} \mathrm{C}$, and combined with $1000 \mu \mathrm{L}$ of warm HEPES solution at $\mathrm{pH}$ 7.4. Samples were incubated at $37^{\circ} \mathrm{C}$ on a rotary shaker and the HEPPES buffer sampled with total replacement after: 30, 60,120 minutes and daily during 8 days showing stable weight. Recovered buffer was soni- 
cated to disrupt residual hydrogel before Uv-vis analysis (Uv-vis Hitachi U-3900). The swelling ratio, DSw, of each type of composite were determined by accurately weighing each gel sample in the stability study after the HEPES buffer had been removed, correcting for residual buffer, and dividing by the original hydrated sample mass [24]-[26]. To compare the degradation of composites with different swelling characteristics, normalized DSw was defined as:

$$
D S w=\frac{m}{m^{*}}
$$

where $m$ and $m^{*}$ are the weight of hydrated composite and dried composite respectively.

\subsection{Drug Release from Biodegradable Hydrogel Composite}

Release profiles of each loaded drug composite were obtained by depositing approximately $30 \mathrm{mg}$ of drug loaded dry hydrogel and adding $1000 \mu \mathrm{L}$ of HEPES buffer into polypropylene sample tubes, warming to $37^{\circ} \mathrm{C}$. Samples were incubated at $37^{\circ} \mathrm{C}$ on a rotary shaker and the HEPPES buffer sampled with total replacement after 15, 30, 60, 120, 360 minutes and $24 \mathrm{~h}$ and daily during 90 days for each preparation. All samples were fresh analyzed by UV-vis measurements were performed on Hitachi U-3900H UV-Vis Spectrophotometer in the wavelength interval of $200-800 \mathrm{~nm}$ at $37^{\circ} \mathrm{C}$ using HEPES buffer as blank. The measurements were accomplished when the values of absorbance of BS in the medium in each sample reached the plateau. These values were transformed to percentage of release $(100 \%)$ and correlated to time (hours) and displayed as time course profile (TC). The fraction of retained water (Wf); hydration percentage $(\% \mathrm{H})$; Swelling (DSw) and time course profile $(\mathrm{t}=100 \%)(\mathrm{TC})$ were evaluated for all hydrogel composite as described in Equations (2)-(4):

$$
\begin{gathered}
W f=\frac{\left(m-m^{*}\right)}{1} \\
E W C=W f \times 100 \\
H=100 \times \frac{\left(m-m^{*}\right)}{m^{*}}
\end{gathered}
$$

where $m$ and $m^{*}$ are the weight of hydrated composite and dried composite respectively.

\subsection{Fourier Transform Infrared (FTIR) Spectroscopy of Biodegradable Hydrogel Composite}

The chemical structure of the biodegradable composite materials and the composite itself (unloaded and loaded with BS) were analyzed by FTIR (IR Prestige-21 FRIT-8400S, Shimadzu, Japan) in transmission mode. For that, each composite $(1.0 \mathrm{mg})$ were mixed with $\mathrm{KBr}(40.0 \mathrm{mg})$ and then formed into a disc in a manual press. Transmission spectra were recorded using at least 32 scans with $4.0 \mathrm{~cm}^{-1}$ resolution, in the spectral range $4000-400 \mathrm{~cm}^{-1}$.

\subsection{Differential Scanning Calorimetry (DSC) Biodegradable Hydrogel Composite}

Thermal characterization of biodegradable hydrogel composite was performed with a Shimadzu DSC-60A. The equipment was calibrated with indium. The sample (approximately $3.0 \mathrm{mg}$ ) was heated twice from $25^{\circ} \mathrm{C}$ to $400^{\circ} \mathrm{C}$ at $20^{\circ} \mathrm{C} / \mathrm{min}$ in a nitrogen atmosphere (flow rate $30 \mathrm{~mL} / \mathrm{min}$ ). The melting temperature $(\mathrm{Tm})$ was determined from the endothermic peak of the DSC curve recorded in the first heating scan.

\subsection{Thermogravimetric Analyses (TGA) of Biodegradable Hydrogel Composite}

The composites and its material were analyses. The samples weighting about $3 \mathrm{mg}$ heated from $25^{\circ} \mathrm{C}$ to $400^{\circ} \mathrm{C}$ in a platinum pan rate $20^{\circ} \mathrm{C} / \mathrm{min}$ in the nitrogen atmosphere at flow of $30 \mathrm{~mL} / \mathrm{min}$. The glass transition temperature (Tg) was determined from the DSC curve recorded in the second heating scan.

\subsection{Scanning Electron Microscopy Measurements (SEM)}

Scanning electron microscopy (SEM) was used to evaluate the surface of biodegradable composite hydrogel. 
Samples containing biodegradable composite hydrogel were mounted on aluminum stubs and due to their lack of electrical conductivity they were coated with $50 \mathrm{~nm}$ gold coating under an argon atmosphere. The composites were examined and photographed by a Jeol 840 A (Tokyo, Japan) Scanning Electron Microscope operating at 20 $\mathrm{kV}$ in the traditional mode (SE1 detector). SEM images were obtained to characterize the surface of the BS-PVA 10\%, BS-GG2\%cys, BS-GG2\%PVA2\%cys and AuNPs-BS-GG2\%PVA2\%cys hydrogel films.

\subsection{Statistical Analysis}

Experiments were performed in triplicate using freshly prepared samples. Statistical analysis data are expressed as means \pm standard deviation unless otherwise noted. Comparisons of groups of means were determined by ANOVA and pairs of mean by Student's t-test where appropriate. Significance was assigned at $\mathrm{p}<0.05$.

\section{Results and Discussion}

\subsection{Hydrogel Composite Preparation}

The first attempt to production of biodegradable hydrogel composite with PVA was done in 7.5\% w/w however, this hydrogel type dissolved when added to solution of HEPES. As alternative, the concentration was increased to $10 \%$. In this way, we characterized the hydrogel in terms of thickness as displayed for GG2\% hydrogel preparation in dried and swollen form (Figure 2) by using a pachymeter. The values obtained for each preparation were GG2\% $0.4 \mathrm{~mm}$ for dry and $3.1 \mathrm{~mm}$ for swollen. Also for PVA10\% were $0.2 \mathrm{~mm}$ dry and 2.0 swollen; GG2\%PVA2\%cys were 0.2 dry and $4.0 \mathrm{~mm}$ swollen and AuNP-GG2\%PVA2\%cys were 0.2 dry and $3.5 \mathrm{~mm}$ swollen.

\subsection{Water Content Parameters and Time Course Release of BS}

Water content in the hydrogel composite was affected by the concentration of gellan gum and also by the cysteine content. The values of the fraction of retained water (Wf); hydration percentage (\%H); Swelling (DSw) is displayed in Table 1. The hydrogel of BS-PVA10\% showed the lower values for all the parameters assayed. Only the time course (TC) of the BS release was higher and this might be due the rigid network during gel formation. Also the others hydrogel composite BS-GG 2\%, BS-GG2\%PVA2\%cys and AuNP-GG2\%PVA2\%cys preparations showed increased values of water parameters with a faster time course of BS release. This results could be explained by the characteristics of GG, a well known polysaccharide manufactured by microbial fermentation of the Sphingomonas paucimobilis microorganism, that can be dissolved in water, and when heated and mixed with mono or divalent cations its forms gels [13]; this behavior associated with PVA and cross-linked with cysteine, turned it in the most suitable composite from the preparations evaluated. These features indicate

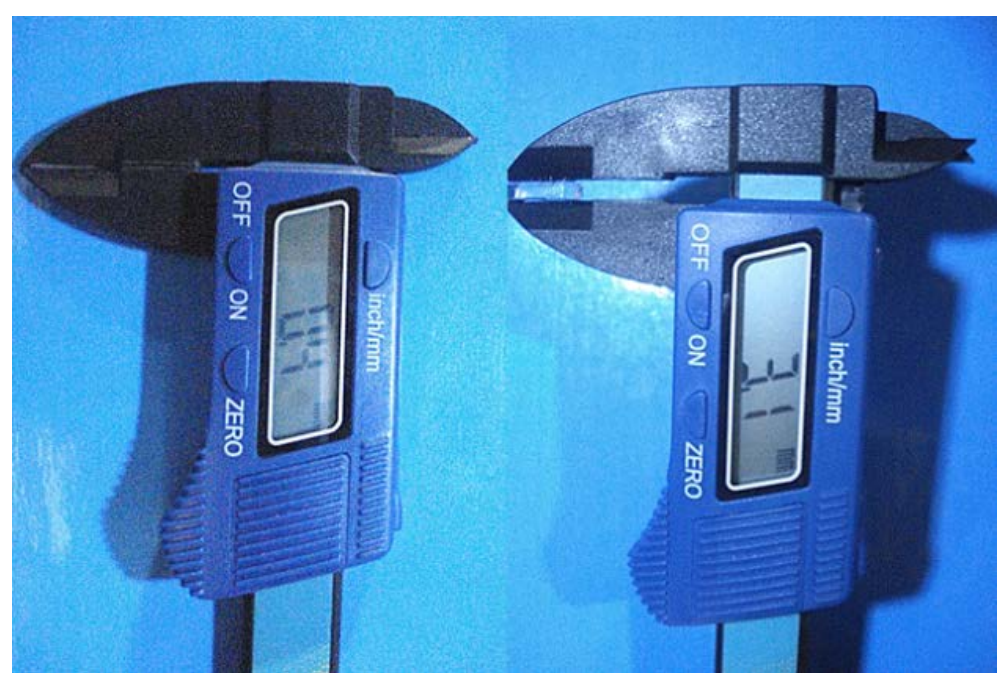

Figure 2. The pachymeter for determining the thickness of GG2\% hydrogel in dried and swollen form. 
Table 1. Biodegradable hydrogel composite: swelling feature and time course release using BS [465 $\mu \mathrm{M}$ ].

\begin{tabular}{ccccc}
\hline \multirow{2}{*}{$\begin{array}{c}\text { Hydrogel composite/ } \\
\text { [BS] }=465 \mu \mathrm{M}\end{array}$} & Wf & Hydrogel properties & TC (hs) \\
\cline { 2 - 5 } & $0.4962 \pm 0.005$ & $96.9 \pm 2.06$ & $1.82 \pm 0.22$ & $72 \pm 3.90$ \\
\hline BS-PVA 10\% & $0.6747 \pm 0.438$ & $211.74 \pm 40.0$ & $3.1174 \pm 0.40$ & $22 \pm 0.04$ \\
BS-GG 2\% & $0.9184 \pm 0.013$ & $1150.87 \pm 186.4$ & $12.508 \pm 1.84$ & $22 \pm 0.15$ \\
BS-GG 2\%PVA2\%cys & $0.9623 \pm 0.021$ & $1450.87 \pm 100.2$ & $8.41 \pm 1.105$ & $14 \pm 0.50$ \\
AuNP-BS-GG2\% PVA2\%cys & &
\end{tabular}

Wf (fraction of retained water); \%H (hydration percentage); DSw (Swelling); TC (Time course).

that this preparation is suitable for a drug delivery system for BS in biological systems with high content of water. The citrate-capped AuNPs decreases the intensity of the crosslinking reaction between the PVA/GGcys and BS showing a less cross-linked hydrogels, with reduced swollen profile when compared with BS-GG2\%PVA $2 \%$ cys.

\subsection{SEM Measurements of Hydrogel Composites}

In Figure 3 are displayed the SEM of the hydrogels surface of BS-PVA 10\%, BS-GG2\%, and BS-GG2\%PVA2\%cys hydrogel films respectively, of dried hydrogels films. The flat and featureless images indicate that the films have a condensed structure and clearly show the appearance of a smooth or fibrous structure. The soft and stiff surface of PVA $10 \%$ can explain the lower time course release of the BS from matrix. However, the porous and fibrous like surface of BS-GG2\%, and BS-GG2\%PVA2\%cys could explain the faster BS release and the increased values of physical chemistry parameters analyzed. In addition, 10\%PVA hydrogel are less able to hydrate than the types with GG2\% explaining its lower time course profile. In these words, GG2\% hydrogels composite and associated with PVA are capable of storage a large amount of BS and maintain longer time at therapeutic line. Both images from Figures 3(B)-(C) displayed more fibrous in topographical surface than the PVA10\%. The AuNP-BS-GG2\%PVA2\%cys displayed in Figure 3(D) shows less fibrous than BS-GG2\%PVA2\%cys due to less crosslink, but due to thermal characteristics of AuNPs embebed into hydrogel, under the experimental conditions the release was faster (36\%) in relation to BS-GG2\%PVA2\%cys alone.

\subsection{FTIR Analysis of Hydrogel Composites}

FTIR spectra was obtained with absorption in the region of $400-4000 \mathrm{~cm}^{-1}$ at room temperature and used to confirm the hydrogels structures. In Figure 4(A) is displayed the PVA 10\% hydrogel FTIR spectra with a characteristic peak at $3343 \mathrm{~cm}^{-1}$ due typical $\mathrm{OH}$ stretching [16]. Also, there is a $2914 \mathrm{~cm}^{-1}$ the asymmetric stretching vibrations of methylene group [24]. At region of $1730 \mathrm{~cm}^{-1}$ it is possible to observe $\mathrm{C}=\mathrm{O}$ stretching [10]. The peak in $1431 \mathrm{~cm}^{-1}$ and $1135 \mathrm{~cm}^{-1}$ indicate the presence of $\mathrm{CH}-\mathrm{OH}$ [27] also in $1381 \mathrm{~cm}^{-1}$ peak is due to $\mathrm{CH}$ asymmetrical bending [26], and in the region of $1095 \mathrm{~cm}^{-1}$ correspond to CO linkage [24]. In Figure 4(B) is displayed the GG2\% hydrogel spectra showing the following peaks in the region $3432 \mathrm{~cm}^{-1}$ that correspond the OH group as shown before in the PVA hydrogel; $1620 \mathrm{~cm}^{-1}$ due to bending vibration of hydroxyl group, 1462 due to $\mathrm{CH}$ asymmetrical bending [28]. In the region of absorption of $1219 \mathrm{~cm}^{-1}$ is possible see the sulfate ion. At the region $1219 \mathrm{~cm}^{-1}$ has a peak that correspond ester linkages [27] and the peak in the $1043 \mathrm{~cm}^{-1}$ indicate the presence of C-O-C group. Both hydrogels of PVA10\% and GG2\% showed preservation of major groups. These characteristics indicate that have no news groups formed in the blend of this hydrogels. It is possible observe, in Figure 4(C) that the GG2\% peaks were preserved as $1620 \mathrm{~cm}^{-1}$ shifted to absorption in $1607 \mathrm{~cm}^{-1}$, ester linkages were also preserved, as show in the $1192 \mathrm{~cm}^{-1}$. This phenomenon is shown in the same graphic with PVA10\% hydrogel peaks. The $\mathrm{C}=\mathrm{O}$ stretching in $1730 \mathrm{~cm}^{-1}$, characteristic peak of this hydrogel, shifted to $1721 \mathrm{~cm}^{-1}$. The infrared spectra did not show absorption bands of the typical bridges resulted from the reaction of the hydrogel and BS [10] [29]. It seem just characteristic bands from BS.

\subsection{Thermal Analysis of Hydrogel Composites (DSC and TGA)}

The DSC heating curves of all hydrogel samples displayed some endothermic peaks due to the melting of the 


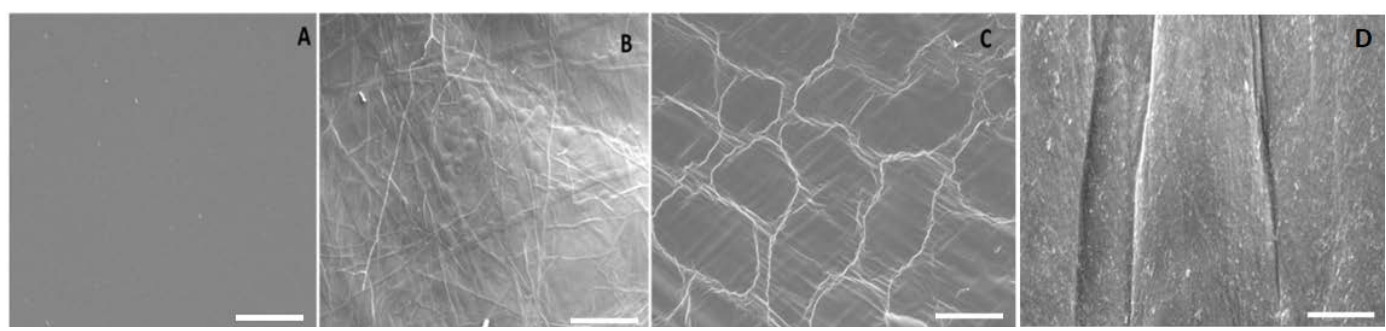

Figure 3. SEM images of hydrogel films. Surface of (a) BS-PVA10\%, (b) BS-GG2\%, (c) BS-GG2\%PVA2\%cys and (d) AuNP-BS-GG2\%PVA2\%cys hydrogel films (270× magnification).

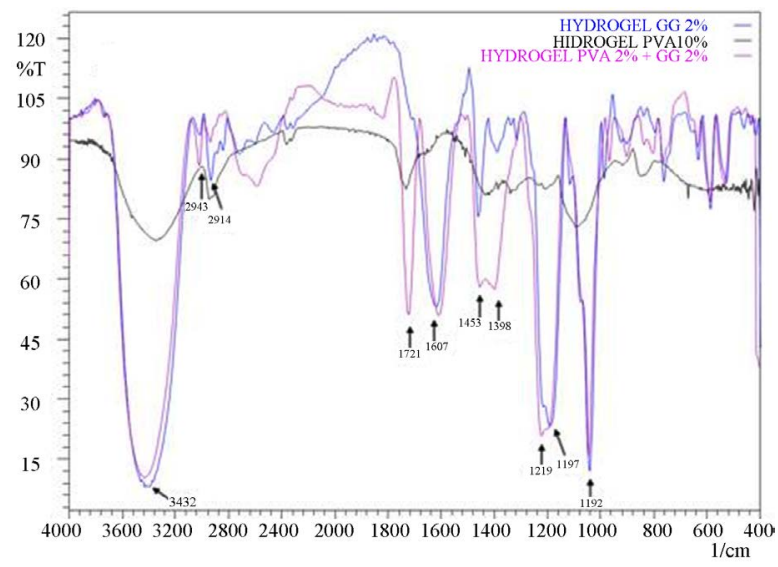

(a)

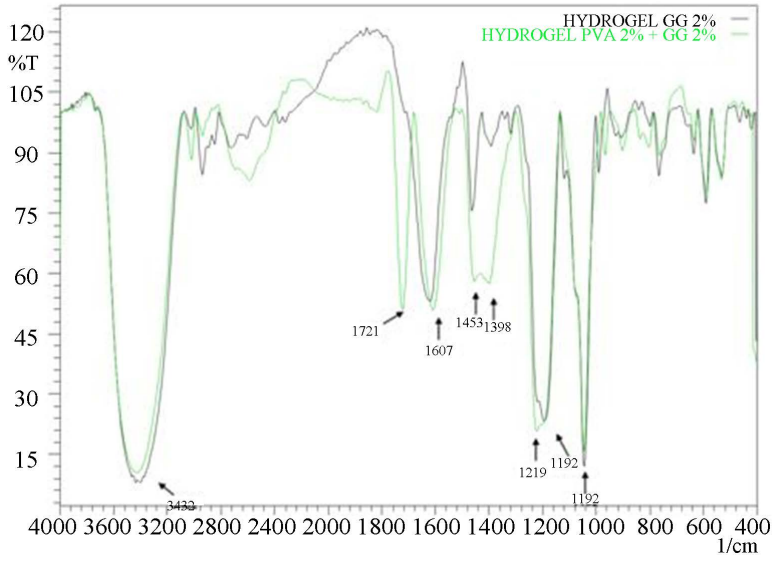

(b)

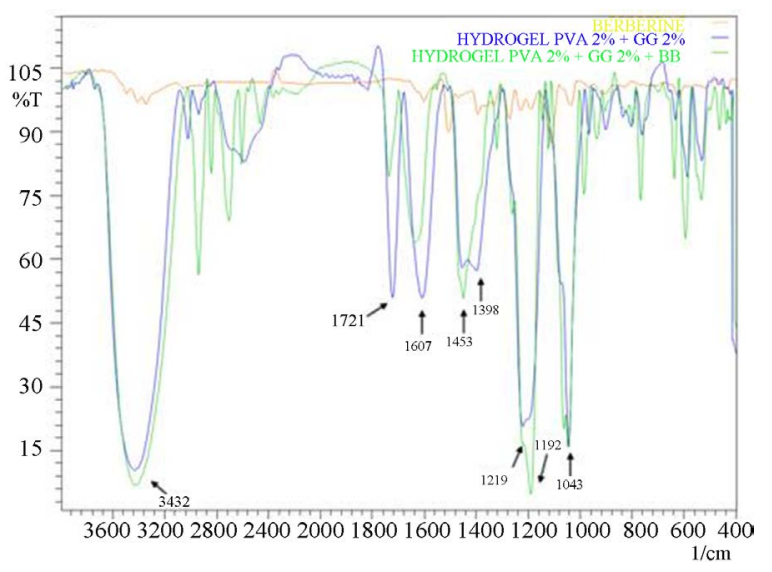

(c)

Figure 4. FTIR spectra of all hydrogel composite: (a) GG2\%; PVA10\%; GG2\%PVA2\%; (b) GG2\% , GG2\%PVA2\%; and (c) BS; GG2\%PVA2\%; BS-GG2\%PVA2\%cys.

various forms of water that freeze during the cooling stage (Figure 5). The endothermic peak around $40^{\circ} \mathrm{C}$ $420^{\circ} \mathrm{C}$ displayed a peak, which have been ascribed previously to the freezing bound water (the sharp one) [30]-[34]. The thermogram is characterized by peaks crystallization (Tc), melting (Tm) and glass transition (Tg) of all hydrogel composite. The behavior observed for 10\%PVA, GG2\%, PVAGG2\% were very similar showing its Tc in the range of $40^{\circ} \mathrm{C}-42^{\circ} \mathrm{C}$ in the first peak and $190^{\circ} \mathrm{C}-260^{\circ} \mathrm{C}$ in the following transitions. The biodegradable hydrogel composite BS-GG2\%PVA2\%cys displayed a similar behavior, however most of the process occurs in exothermic profile. When association occurs, the formation of more structured complex so formed exhibits different characteristics in comparison to components-alone. AuNPs-BS-GG2\%PVA2\%cys showed similar behavior

In Figure 6 is displayed the normalized TGA measurements of all components used in the synthesis biode- 


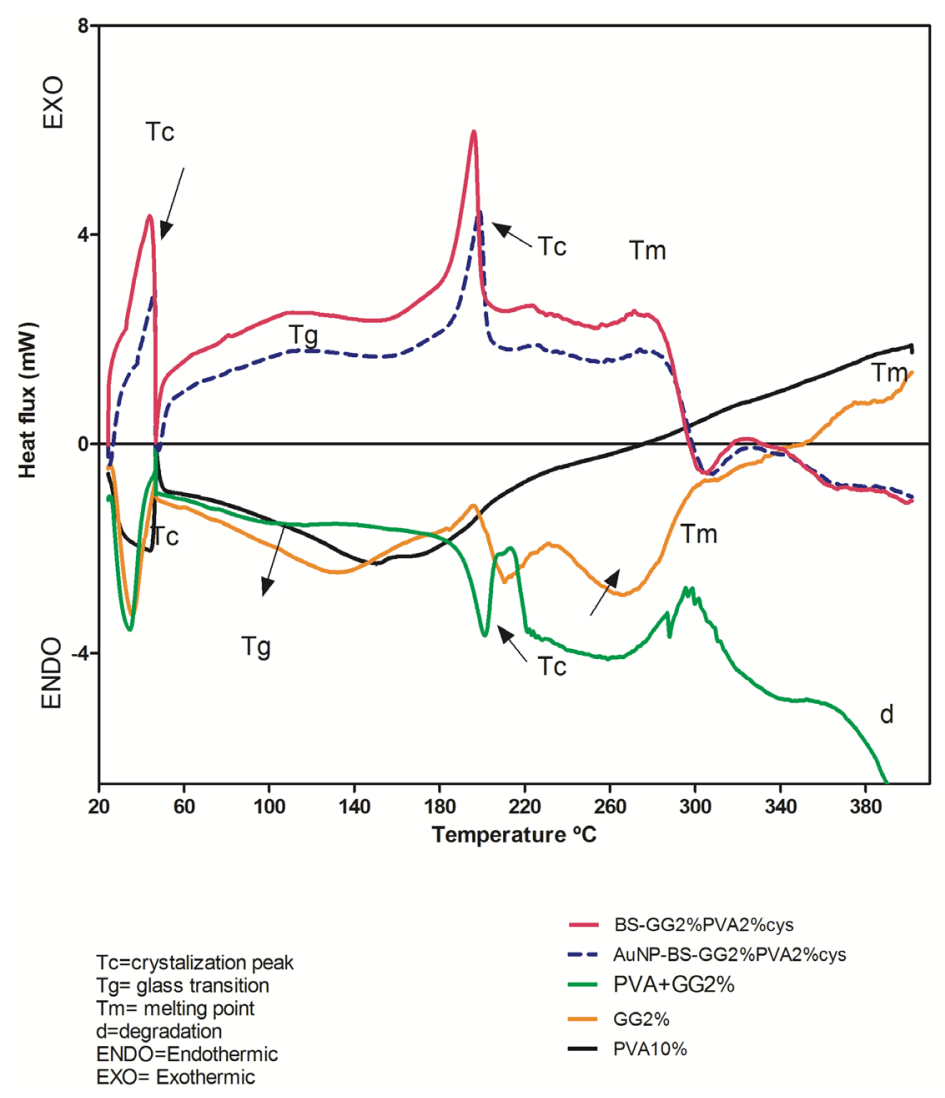

Figure 5. DSC spectra of hydrogel composite: PVA 10\%; GG2 \%; GG2\%PVA2\%cys; BS-GG2\%PVA2\%cys and AuNP-BS-GG2\%PVA2\%cys.

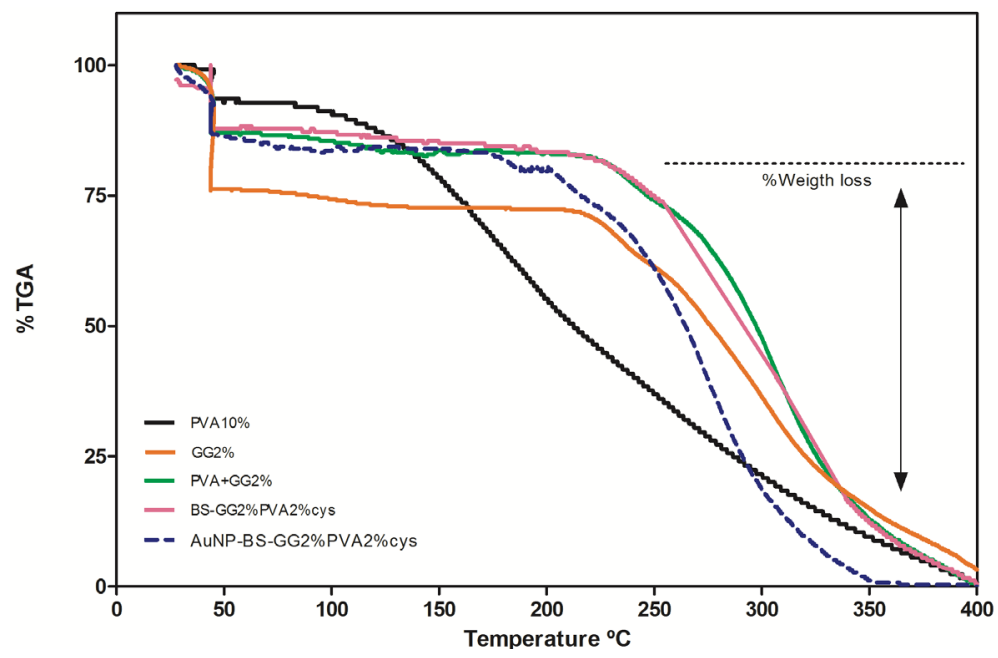

Figure 6. TGA spectra of hydrogel composite: PVA 10\%; GG2 \%; GG2\% PVA2\%cys; BS-GG2\%PVA2\%cys and AuNP-BS-GG2\%PVA2\%cys.

gradable hydrogel composite. The evaporation process occurs more drastically in the GG2\%-(23.0\%) and the other component were respectively PVA10\%-(5.02\%); PVAGG2\%-(12.1\%); BS-GG2\%PVA2\%cys-(10.2\%) and AuNP-BS-GG2\%PVA2\%cys-(12.1\%). These results indicated that GG2\% hydrogel lose more water than PVA10\%, also when it is incorporated to PVA and the biodegradable hydrogel loaded with BS, this water lost is reduced indicating that the structure is modified entrapping the water inside the system. The percentage weight 
loss and the start temperature for all formulation were GG2\% $\left(71.5 \%\right.$ at $\left.214^{\circ} \mathrm{C}\right)$; PVA10\%-( $90 \%$ at $\left.100^{\circ} \mathrm{C}\right)$; PVAGG2\%-(81.4\% at $\left.225^{\circ} \mathrm{C}\right)$; BS-GG2\%PVA2\%cys-(81.2\% at $\left.225^{\circ} \mathrm{C}\right)$ and AuNP-BS-GG2\%PVA2\%cys-(80\% at $215^{\circ} \mathrm{C}$ ) were observed for all system These results indicate that the GG2\% has less mass to lose since its structure is less porous than the network achieved with PVAGG2\% BS-GG2\%PVA2\%cys and AuNP-BSGG2\%PVA2\%cys.

\section{Conclusions}

Polymeric drug delivery system allows the development of innovative concepts that could create more efficient systems to achieve a prolonged and controlled drug delivery. In this study, by the use of the crosslink method, we have prepared several hydrogel composite by varying amount of GG and PVA loaded with BS and its association to gold nanoparticles, and then characterized its water holding capacities, time course release profile; physical-chemical behavior (FTIR, DSC; TGA) and surface analysis (SEM).

The results have demonstrated distinct surface profile of the hydrogel composites and two different time course profiles, which could explain by the feature of hydrogel matrix.

Among all biodegradable hydrogel composites evaluated it was clear that the addition of GG associated to PVA and association to gold nanoparticle, produced the most suitable drug delivery system for BS, due to all characteristics (higher entrapment efficiency) of the matrix structure, making a promising drug delivery system for delivery hydrophilic drugs. Future application of gold nanoparticles (AuNPs) added into the transparent loaded hydrogel can be a promising efficient drug delivery system in skin, by enabling the cell activating capacity once its size is only $1 / 2000$ as large as pores, very easy to penetrate into the dermis. It can carry the drug such as BS deep into the skin to activate skin cells, increases vasodilatation and can enhance the compactness of the fibrous tissue of collagen to turn the skin compact and elastic against aging and other process.

\section{Acknowledgements}

This material is based upon work supported by the CNPq, FAPDF, FINATEC, DPP-UnB, CAPES.

\section{References}

[1] Huang, Z.J., Zeng, Y., Lan, P., Sun, P.H. and Chen, W.M. (2011) Advances in Structural Modifications and Biological Activities of Berberine: An Active Compound in Traditional Chinese Medicine. Mini-Reviews in Medicinal Chemistry, 11, 1122-1129. http://dx.doi.org/10.2174/138955711797655362

[2] Tillhon, M., Guamán Ortiz, L.M., Lombardi, P. and Scovassi, A.I. (2012) Berberine: New Perspectives for Old Remedies. Biochemical Pharmacologyhem Pharmacol, 84, 1260-1267. http://dx.doi.org/10.1016/j.bcp.2012.07.018

[3] Xie, W. and Du, L. (2011) Diabetes Is an Inflammatory Disease: Evidence from Traditional Chinese Medicines. Diabetes, Obesity and Metabolism, 13, 289-301. http://dx.doi.org/10.1111/j.1463-1326.2010.01336.x

[4] Wang, X.H., Jiang, S.M. and Sun, Q.W. (2011) Effects of Berberine on Human Rheumatoid Arthritis Fibroblast-Like Synoviocytes. Experimental Biology and Medicine (Maywood), 236, 859-866. http://dx.doi.org/10.1258/ebm.2011.010366

[5] Chen, X.W., Di, Y.M., Zhang, J., Zhou, Z.W., Li, C.G. and Zhou, S.F. (2012) Interaction of Herbal Compounds with Biological Targets: A Case Study with Berberine. Scientific World Journal, 2012, Article ID: 708292. http://dx.doi.org/10.1100/2012/708292

[6] Chen, Y., Wang, Y., Zhang, J., Sun, C. and Lopez, A. (2011) Berberine Improves Glucose Homeostasis in Streptozotocin-Induced Diabetic Rats in Association with Multiple Factors of Insulin Resistance. ISRN Endocrinology, 2011, Article ID: 519371. http://dx.doi.org/10.5402/2011/519371

[7] Chidambara Murthy, K.N., Jayaprakasha, G.K. and Patil, B.S. (2012) The Natural Alkaloid Berberine Targets Multiple Pathways to Induce Cell Death in Cultured Human Colon Cancer Cells. European Journal of Pharmacology, 688, 1421. http://dx.doi.org/10.1016/j.ejphar.2012.05.004

[8] Tan, W., Li, Y., Chen, M. and Wang, Y. (2011) Berberine Hydrochloride: Anticancer Activity and Nanoparticulate Delivery System. International Journal of Nanomedicine, 6, 1773-1777. http://dx.doi.org/10.2147/IJN.S22683

[9] Trimarco, V., Cimmino, C.S., Santoro, M., Pagnano, G., Manzi, M.V., Piglia, A., et al. (2012) Nutraceuticals for Blood Pressure Control in Patients with High-Normal or Grade 1 Hypertension. High Blood Pressure \& Cardiovascular Prevention, 19, 117-122.

[10] Wang, J., Liu, Q. and Yang, Q. (2012) Radiosensitization Effects of Berberine on Human Breast Cancer Cells. Interna- 
tional Journal of Molecular Medicine, 30, 1166-1172.

[11] Wang, Y., Huang, Y., Lam, K.S., Li, Y., Wong, W.T., Ye, H., et al. (2009) Berberine Prevents Hyperglycemia-Induced Endothelial Injury and Enhances Vasodilatation via Adenosine Monophosphate-Activated Protein Kinase and Endothelial Nitric Oxide Synthase. Cardiovascular Research, 82, 484-492. http://dx.doi.org/10.1093/cvr/cvp078

[12] Zhou, L., Wang, X., Yang, Y., Wu, L., Li, F., Zhang, R., et al. (2011) Berberine Attenuates cAMP-Induced Lipolysis via Reducing the Inhibition of Phosphodiesterase in 3T3-L1 Adipocytes. Biochimica et Biophysica Acta (BBA)—Molecular Basis of Disease, 1812, 527-535. http://dx.doi.org/10.1016/j.bbadis.2010.10.001

[13] Minhas, M.U., Ahmad, M., Ali, L. and Sohail, M. (2013) Synthesis of Chemically Cross-Linked Polyvinyl Alcohol-coPoly (methacrylic acid) Hydrogels by Copolymerization; A Potential Graft-Polymeric Carrier for Oral Delivery of 5Fluorouracil. DARU Journal of Pharmaceutical Sciences, 21. http://dx.doi.org/10.1186/2008-2231-21-44

[14] Zhang, X.Z., Zhuo, R.X., Cui, J.Z. and Zhang, J.T. (2002) A Novel Thermo-Responsive Drug Delivery System with Positive Controlled Release. International Journal of Pharmaceutics, 235, 43-50. http://dx.doi.org/10.1016/S0378-5173(01)00976-0

[15] Liu, L., Wang, B., Gao, Y. and Bai, T.C. (2013) Chitosan Fibers Enhanced Gellan Gum Hydrogels with Superior Mechanical Properties and Water-Holding Capacity. Carbohydrate Polymers, 97, 152-158. http://dx.doi.org/10.1016/j.carbpol.2013.04.043

[16] Moura, M.J., Faneca, H., Lima, M.P., Gil, M.H. and Figueiredo, M.M. (2011) In Situ Forming Chitosan Hydrogels Prepared via Ionic/Covalent Co-Cross-Linking. Biomacromolecules, 12, 3275-3284. http://dx.doi.org/10.1021/bm200731x

[17] Cencetti, C., Bellini, D., Pavesio, A., Senigaglia, D., Passariello, C., Virga, A., et al. (2012) Preparation and Characterization of Antimicrobial Wound Dressings Based on Silver, Gellan, PVA and Borax. Carbohydrate Polymers, 90, 1362-1370. http://dx.doi.org/10.1016/j.carbpol.2012.07.005

[18] Cobley, C.M., Chen, J., Cho, E.C., Wang, L.V. and Xia, Y. (2011) Gold Nanostructures: A Class of Multifunctional Materials for Biomedical Applications. Chemical Society Reviews, 40, 44-56. http://dx.doi.org/10.1039/b821763g

[19] Bedford, E.E., Spadavecchia, J., Pradier, C.M. and Gu, F.X. (2012) Surface Plasmon Resonance Biosensors Incorporating Gold Nanoparticles. Macromolecular Bioscience, 12, 724-739. http://dx.doi.org/10.1002/mabi.201100435

[20] Cristallini, C., Guerra, G.D., Barbani, N. and Bianchi, F. (2007) Biodegradable Bioartificial Materials Made with Chitosan and Poly(vinyl alcohol). Part I: Physicochemical Characterization. Journal of Applied Biomaterials \& Biomechanics, 5, 184-191.

[21] Cascone, M.G., Barbani, N., Cristallini, C., Giusti, P., Ciardelli, G. and Lazzeri, L. (2001) Bioartificial Polymeric Materials Based on Polysaccharides. Journal of Biomaterials Science, Polymer Edition, 12, 267-281. http://dx.doi.org/10.1163/156856201750180807

[22] Oliveira, J.T., Martins, L., Picciochi, R., Malafaya, P.B., Sousa, R.A., Neves, N.M., et al. (2010) Gellan Gum: A New Biomaterial for Cartilage Tissue Engineering Applications. Journal of Biomedical Materials Research Part A, 93, 852863.

[23] Kimling, J., Maier, M., Okenve, B., Kotaidis, V., Ballot, H. and Plech, A. (2006) Turkevich Method for Gold Nanoparticle Synthesis Revisited. Journal of Physical Chemistry B, 110, 15700-15707. http://dx.doi.org/10.1021/jp061667w

[24] Cai, X., Shao, W., Luan, Y., Pang, J., Li, F. and Li, Z. (2011) Metformin Hydrochloride-Loaded Poly(vinyl alcohol) Composites as Drug Delivery Systems. Journal of Nanoscience and Nanotechnology, 11, 8621-8627. http://dx.doi.org/10.1166/jnn.2011.4762

[25] Chen, C.H., Wang, F.Y., Mao, C.F., Liao, W.T. and Hsieh, C.D. (2008) Studies of Chitosan: II. Preparation and Characterization of Chitosan/Poly(vinyl alcohol)/Gelatin Ternary Blend Films. International Journal of Biological Macromolecules, 43, 37-42. http://dx.doi.org/10.1016/j.ijbiomac.2007.09.005

[26] Lima, A.C., Sher, P. and Mano, J.F. (2012) Production Methodologies of Polymeric and Hydrogel Particles for Drug Delivery Applications. Expert Opinion on Drug Delivery, 9, 231-248. http://dx.doi.org/10.1517/17425247.2012.652614

[27] Hu, Y., Wang, Q. and Tang, M. (2013) Preparation and Properties of Starch-g-PLA/Poly(vinyl alcohol) Composite Film. Carbohydrate Polymers, 96, 384-388. http://dx.doi.org/10.1016/j.carbpol.2013.04.011

[28] Krauland, A.H., Leitner, V.M. and Bernkop-Schnürch, A. (2003) Improvement in the in Situ Gelling Properties of Deacetylated Gellan Gum by the Immobilization of Thiol Groups. Journal of Pharmaceutical Sciences, 92, 1234-1241. http://dx.doi.org/10.1002/jps.10371

[29] Bashmakova, N., Kutovyy, S., Kornienko, M., Yashchuk, V., Hovorun, D. and Zhurakivsky, R. (2010) Experimental and Calculated by the DFT Method Vibration Spectra of Berberine. Xxii International Conference on Raman Spectroscopy, 1267, 426-427. 
[30] Ahuja, M., Singh, S. and Kumar, A. (2013) Evaluation of Carboxymethyl Gellan Gum as a Mucoadhesive Polymer. International Journal of Biological Macromolecules, 53, 114-121. http://dx.doi.org/10.1016/j.ijbiomac.2012.10.033

[31] Coutinho, D.F., Sant, S.V., Shin, H., Oliveira, J.T., Gomes, M.E., Neves, N.M., et al. (2010) Modified Gellan Gum Hydrogels with Tunable Physical and Mechanical Properties. Biomaterials, 31, 7494-7502. http://dx.doi.org/10.1016/j.biomaterials.2010.06.035

[32] Liu, Y., Geever, L.M., Kennedy, J.E., Higginbotham, C.L., Cahill, P.A. and McGuinness, G.B. (2010) Thermal Behavior and Mechanical Properties of Physically Crosslinked PVA/Gelatin Hydrogels. Journal of the Mechanical Behavior of Biomedical Materials, 3, 203-209. http://dx.doi.org/10.1016/j.jmbbm.2009.07.001

[33] Peng, Z., Kong, L.X., Li, S.D. and Spiridonov, P. (2006) Poly(vinyl alcohol)/Silica Nanocomposites: Morphology and Thermal Degradation Kinetics. Journal of Nanoscience and Nanotechnology, 6, 3934-3938. http://dx.doi.org/10.1166/jnn.2006.666

[34] Wang, H.Y., Lu, S.S. and Lun, Z.R. (2009) Glass Transition Behavior of the Vitrification Solutions Containing Propanediol, Dimethyl Sulfoxide and Polyvinyl Alcohol. Cryobiology, 58, 115-117. http://dx.doi.org/10.1016/j.cryobiol.2008.10.131 
Scientific Research Publishing (SCIRP) is one of the largest Open Access journal publishers. It is currently publishing more than 200 open access, online, peer-reviewed journals covering a wide range of academic disciplines. SCIRP serves the worldwide academic communities and contributes to the progress and application of science with its publication.

Other selected journals from SCIRP are listed as below. Submit your manuscript to us via either submit@scirp.org or Online Submission Portal.
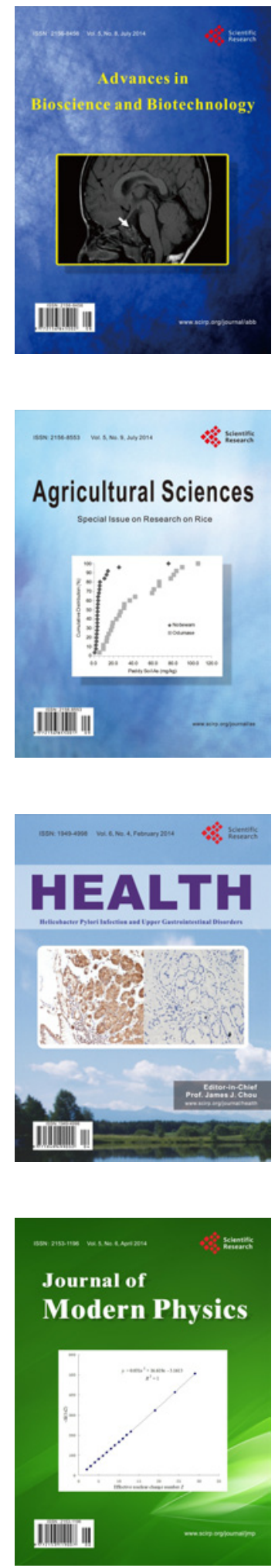
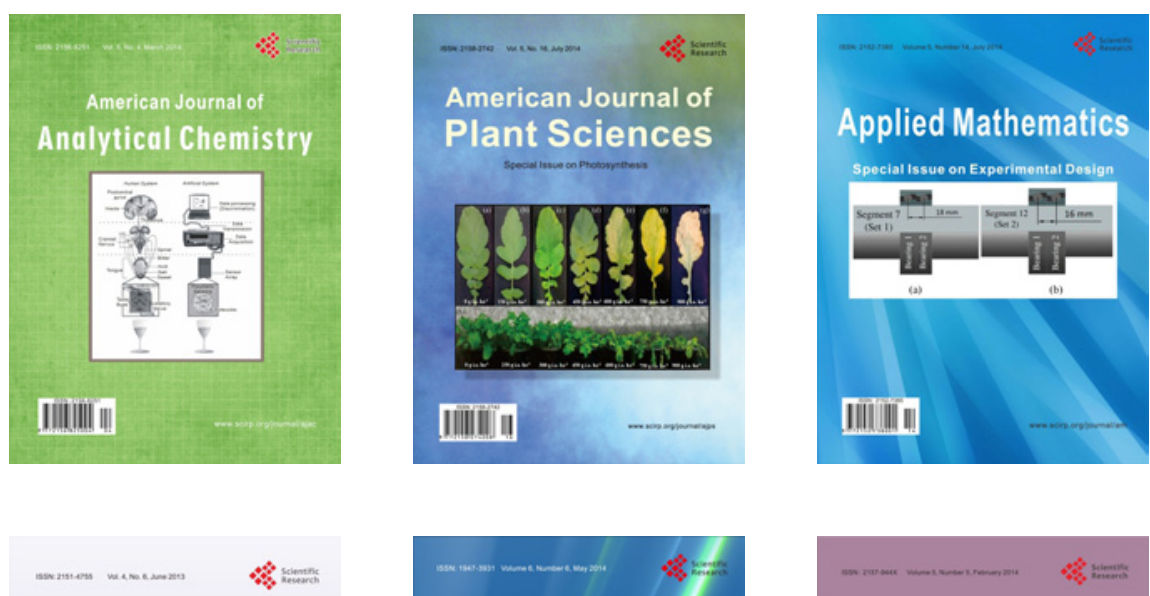

Creative Education
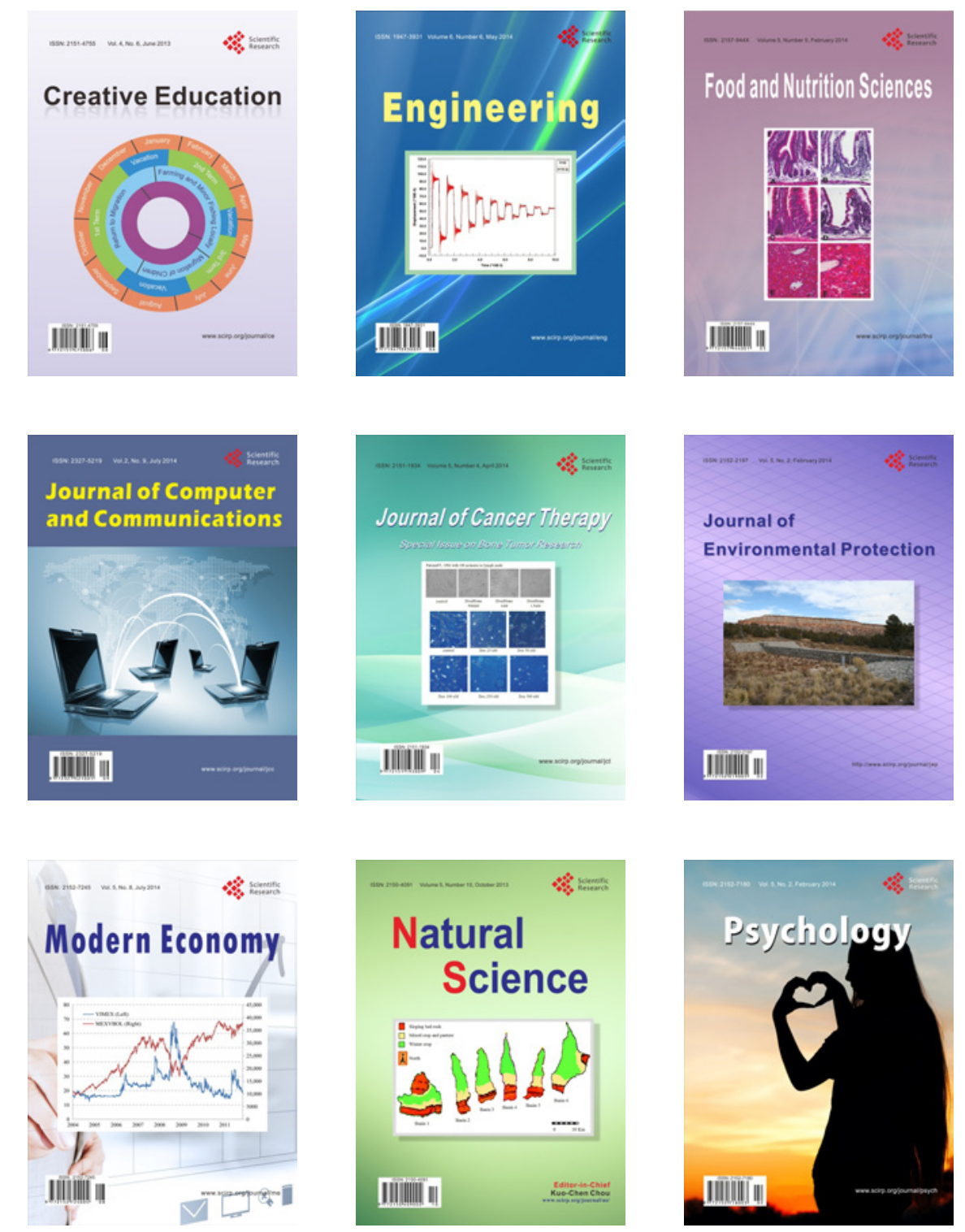